NASA/TM-2002-211503

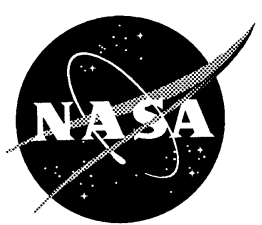

\title{
Laser Light Scattering Diagnostic for Measurement of Flow Velocity in Vicinity of Propagating Shock Waves
}

Richard G. Seasholtz and Alvin E. Buggele

Glenn Research Center, Cleveland, Ohio 
The NASA STI Program Office ... in Profile

Since its founding, NASA has been dedicated to the advancement of aeronautics and space science. The NASA Scientific and Technical Information (STI) Program Office plays a key part in helping NASA maintain this important role.

The NASA STI Program Office is operated by Langley Research Center, the Lead Center for NASA's scientific and technical information. The NASA STI Program Office provides access to the NASA STI Database, the largest collection of aeronautical and space science STI in the world. The Program Office is also NASA's institutional mechanism for disseminating the results of its research and development activities. These results are published by NASA in the NASA STI Report Series, which includes the following report types:

- TECHNICAL PUBLICATION. Reports of completed research or a major significant phase of research that present the results of NASA programs and include extensive data or theoretical analysis. Includes compilations of significant scientific and technical data and information deemed to be of continuing reference value. NASA's counterpart of peerreviewed formal professional papers but has less stringent limitations on manuscript length and extent of graphic presentations.

- TECHNICAL MEMORANDUM. Scientific and technical findings that are preliminary or of specialized interest, e.g., quick release reports, working papers, and bibliographies that contain minimal annotation. Does not contain extensive analysis.

- CONTRACTOR REPORT. Scientific and technical findings by NASA-sponsored contractors and grantees.
- CONFERENCE PUBLICATION. Collected papers from scientific and technical conferences, symposia, seminars, or other meetings sponsored or cosponsored by NASA.

- SPECIAL PUBLICATION. Scientific, technical, or historical information from NASA programs, projects, and missions, often concerned with subjects having substantial public interest.

- TECHNICAL TRANSLATION. Englishlanguage translations of foreign scientific and technical material pertinent to NASA's mission.

Specialized services that complement the STI Program Office's diverse offerings include creating custom thesauri, building customized data bases, organizing and publishing research results ... even providing videos.

For more information about the NASA STI Program Office, see the following:

- Access the NASA STI Program Home Page at http://www.sti.nasa.gov

- E-mail your question via the Internet to help@sti.nasa.gov

- Fax your question to the NASA Access Help Desk at 301-621-0134

- Telephone the NASA Access Help Desk at 301-621-0390

- Write to: NASA Access Help Desk NASA Center for AeroSpace Information 7121 Standard Drive Hanover, MD 21076 
NASA/TM-2002-211503

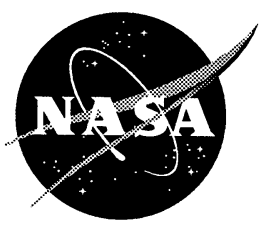

Laser Light Scattering Diagnostic for Measurement of Flow Velocity in Vicinity of Propagating Shock Waves

Richard G. Seasholtz and Alvin E. Buggele

Glenn Research Center, Cleveland, Ohio

Prepared for the

2001 19th International Congress on Instrumentation in Aerospace Simulation Facilities (ICIASF 2001)

cosponsored by the IEEE AES, NASA Glenn, and OAI

Cleveland, Ohio, August 27-30, 2001

National Aeronautics and

Space Administration

Glenn Research Center 


\section{Acknowledgments}

We would like to acknowledge the efforts of Mr. Raymond Lotenero, who was responsible for setting up and aligning the optical system used for this work, and Ms. Kristie Elam, who developed the data acquisition system.

Available from

NASA Center for Aerospace Information

7121 Standard Drive

National Technical Information Service Hanover, MD 21076 5285 Port Royal Road Springfield, VA 22100 


\title{
LASER LIGHT SCATTERING DIAGNOSTIC FOR MEASUREMENT OF FLOW VELOCITY IN VICINITY OF PROPAGATING SHOCK WAVES
}

\author{
Richard G. Seasholtz and Alvin E. Buggele \\ National Aeronautics and Space Administration \\ Glenn Research Center \\ Cleveland, Ohio 44135
}

\begin{abstract}
A laser light scattering diagnostic for measurement of dynamic flow velocity at a point is described. The instrument is being developed for use in the study of propagating shock waves and detonation waves in pulse detonation engines under development at the NASA Glenn Research Center (GRC). The approach uses a Fabry-Perot interferometer to measure the Doppler shift of laser light scattered from small (submicron) particles in the flow. The high-speed detection system required to resolve the transient response as a shock wave crosses the probe volume uses fast response photodetectors and a PC based data acquisition system. Preliminary results of measurements made in the GRC Mach 4, 10x25 cm supersonic wind tunnel are presented. Spontaneous condensation of water vapor in the flow is used as seed. The tunnel is supplied with continuous air flow at up to $45 \mathrm{psia}$; the flow is exhausted into the GRC laboratory-wide altitude exhaust system at pressures down to 0.3 psia.
\end{abstract}

\section{INTRODUCTION}

In this paper we describe a point measurement flow diagnostic being developed for high frequency response measurements of flow velocity. The instrument is being developed for use in the study of propagating shock waves and detonation waves in pulse detonation engines under development at the NASA Glenn Research Center. Laser based flow diagnostics for high frequency response measurements include Laser Doppler Velocimetry (LDV), which is typically based on scattering from particles in the one micrometer size range. In addition to concerns about particle lag, it is difficult to obtain data rates in excess of about $10 \mathrm{kHz}$. In turbulent flows, LDV measurements are beset by a variety of socalled biasing errors, caused by correlations between the measurement rate and flow properties. Other approaches, including the work presented here, are based on scattering from a large concentration of particles in the submicrometer size range. These techniques are based on measuring the Doppler shift of the scattered light using some type of frequency discriminator to convert changes in the optical frequency of the scattered light to changes in intensity. Discriminators that have been used include a Michelson interferometer ${ }^{1}$, a confocal Fabry-Perot interferometer $^{2}$ and planar mirror Fabry-Perot interferometers ${ }^{3}$. Another type of optical frequency discriminator used for velocity measurement is based on atomic or molecular absorption cells ${ }^{4,5}$. However, this approach requires use of a laser that can be tuned to the absorption line being used.

The approach we use is based on a previously developed system to measure flow density and velocity based on molecular Rayleigh scattering. The frequency discriminator is a planar mirror Fabry-Perot interferometer used in the static, imaging mode. Unfortunately, molecular Rayleigh scattering using CW lasers in the 1-5 $\mathrm{W}$ range normally does not provide sufficiently strong scattering to allow high frequency response transient measurements. The alternative is to introduce artificial seed material into the flow to provide more intense scattering than that provided by the molecules that make up the actual flow. One approach for producing seed is to use a vaporizationcondensation aerosol generator. This seed can then be introduced into the flow upstream of the measurement location. Another approach that can be used in flow undergoing expansion (and resulting cooling) is to rely on a condensable vapor. Water or carbon dioxide, for example, will form an aerosol by spontaneous condensation as the flow expands. This is the approach used in this paper. However, as we show, this does not provide sufficient scattering for high Mach number flows.

Preliminary results of measurements made in the GRC Mach 4, 10x25 cm supersonic wind tunnel are presented. For these measurements, a beam from a $5 \mathrm{~W}$ $\mathrm{CW}$ single-frequency $\mathrm{Nd}$ :Vanadate laser was focused at a point in the flow. Scattered light was collected and transmitted over $130 \mathrm{~m}$ of optical fiber to a remote location, where the Fabry-Perot interferometer, photodetectors, and PC based data acquisition system were located. 
Specific issues addressed include the design of a baffle assembly to allow the laser beam to pass through the wind tunnel test section without introducing excessive laser light scattering into the receiving optics. Also, the adequacy of using spontaneous condensation of water vapor as a seed material is examined. It is shown that spontaneous condensation of water vapor in ambient temperature air supplied to a relatively high Mach number (Mach 4) supersonic nozzle does not provide adequate scattering for $100 \mathrm{kHz}$ sampling rate measurements.

\section{Light Scattering}

\section{THEORY} object is

The frequency shift of light scattered from a moving

$$
f_{s}=f-f_{o} \quad \frac{\mathbf{K} \cdot \mathbf{u}}{2 \pi}
$$

where $f_{0}$ is the laser frequency and $\mathbf{u}$ is the particle velocity. The interaction wave vector is $\mathbf{K}=\mathbf{k}_{\mathrm{s}}-\mathbf{k}_{\mathrm{o}}$ (with $\mathbf{k}_{\mathbf{o}}$ and $\mathbf{k}_{\mathrm{s}}$ being the wave vectors of the incident and scattered light. It is convenient to introduce the velocity component $u_{\mathrm{K}}=\mathbf{K} \cdot \mathbf{u} /|\mathbf{K}|$, which represents the measured velocity component.

The intensity of the detected scattered light can be expressed in terms of photoelectron count rate

$$
N_{R}=\frac{\varepsilon P_{o} n_{s} L_{x} \lambda \Omega}{h c}\left(\frac{\mathrm{d} \sigma}{\mathrm{d} \Omega}\right) \sin ^{2} \chi
$$

where $\varepsilon$ is the efficiency factor (which includes the detector quantum efficiency), $P_{o}$ is the laser power, $n_{s}$ is number density of the scattering particles, $L_{x}$ is the length of the probe volume, $\lambda$ is the laser wavelength, $\Omega$ is the solid angle of the light collection optics, $\mathrm{d} \sigma / \mathrm{d} \Omega$ is the particle differential scattering cross section, $\chi$ is the angle between the electric field vector of the (linearly polarized) incident light and the direction of the scattered light, $h$ is Planck's constant, and $c$ is the velocity of light. For the work presented here we assume that $\sin ^{2} \chi=1$, which corresponds to "s" type scattering.

Two factors determine the amount of scattering light that is available for making a measurement. One is the fraction of the total scattering light that can be collected and analyzed. This is limited by the collection optics and/or other optical components in the system. It is convenient to use the Lagrange Invariant to express this quantity. In our system, the optical fiber used to transmit the scattered light from the test cell to the optical processing area sets the limit on the amount of available scattered light. The Lagrange Invariant for the fiber is

$$
A \Omega=\frac{\pi D_{f}^{2}}{4} \pi N A^{2}
$$

where $D_{f}$ is the core diameter of the fiber and $N A$ is its numerical aperture. For our system, $D_{f}=0.55 \mathrm{~mm}$ and $N A=0.22$, so $A \Omega=0.036 \mathrm{~mm}^{2}$-sr. Given the f-number of the collection optics, we can determine the probe volume size using the value of the Lagrange Invariant set by the fiber.

$$
A \Omega=\frac{\pi L_{x}^{2}}{4} \frac{\pi}{4(f / \#)^{2}}
$$

Thus, for $\mathrm{f} / 4$ collection optics, the probe volume length $\mathrm{L}_{\mathrm{x}}=0.97 \mathrm{~mm}$.

The other factor that determines the amount of scattered light that is available for making a measurement is the product of the number density of scattering particles $n_{s}$ and their differential scattering cross section $(\mathrm{d} \sigma / \mathrm{d} \Omega)$. To determine the quantity $n_{s}(\mathrm{~d} \sigma / \mathrm{d} \Omega)$ we need to establish the size and concentration of the seed.

\section{Seeding}

To achieve high frequency response measurements, the flow must contain scattering centers in sufficiently high numbers so the intensity of the scattering will allow measurements with the desired uncertainty. With weak scattering, the primary source of error is photon statistical noise (shot noise). A measure of the scattering efficiency is the product of the number density of scattering centers $n_{s}$ and the differential scattering cross section $(\mathrm{d} \sigma / \mathrm{d} \Omega)$. As a reference point, molecular Rayleigh scattering of $532 \mathrm{~nm}$ light from air at NPT (normal pressure and temperature) has $n_{s}=2.5 \times 10^{25} \mathrm{~m}^{-3}$ and $(\mathrm{d} \sigma / \mathrm{d} \Omega)=$ $6.1 \times 10^{-32} \mathrm{~m}^{2} \mathrm{sr}^{-1}$, so $n_{s}(\mathrm{~d} \sigma / \mathrm{d} \Omega)=1.5 \times 10^{-6} \mathrm{~m}^{-1} \mathrm{sr}^{-1}$. For submicron size particles, the scattering cross section is proportional to the sixth power of the diameter, and the number density of particles for a fixed amount of material is proportional to the inverse cube of the diameter. Thus the scattering efficiency is proportional to the cube of the particle diameter for a given amount of material. The optimum particle size is thus the largest particle that will follow the flow acceleration. Of course, the particles must also have a large enough number density so that there will be one or more particles in the probe volume during each sampling interval.

As an example, consider the water vapor in air with $100 \%$ relative humidity at $20^{\circ} \mathrm{C}$, which has a partial pressure of 0.34 psia. If we expand this humid air through a supersonic nozzle, most of the water vapor 
will condense into liquid water droplets or ice particles. The differential scattering cross section is shown on figure 1 for a refractive index of 1.3 at $0.532 \mu \mathrm{m}$ wavelength. Figure 2 shows the quantity $n_{s}(\mathrm{~d} \sigma / \mathrm{d} \Omega)$ for Mach 2 and Mach 4 expansion. The size distribution of droplets formed by spontaneous condensation depends on the particular parameters of the expansion ${ }^{7}$. As an example, consider Mach 4 flow, and assume a particle diameter of $0.050 \mu \mathrm{m}$. Then the number density of scattering centers will be about $n_{s}=0.5 \times 10^{13} \mathrm{~m}^{-3}$, $(\mathrm{d} \sigma / \mathrm{d} \Omega)=1.7 \times 10^{-19} \mathrm{~m}^{2} \mathrm{sr}^{-1}$ and the product $n_{s}(\mathrm{~d} \sigma / \mathrm{d} \Omega)=$ $9 \times 10^{-7} \mathrm{~m}^{-1} \mathrm{sr}^{-1}$. We can then use equation 1 with $P_{o}=$ $5 \mathrm{~W}, \varepsilon=5 \%, L_{x}=1 \mathrm{~mm}, \Omega=0.05 \mathrm{sr}$, and $\lambda=0.532$ $\mu \mathrm{m}$. to calculate the photoelectron count rate, $N_{R}=3 \mathrm{x}$ $10^{7}$ counts/sec. Although, this may seem to be a rather large rate, the number of counts in a $100 \mu \mathrm{sec}$ interval is only 300 , and the uncertainty due the photon statistical noise is about $300^{-1 / 2}$, or $6 \%$. This is marginal for even measuring the amplitude of the signal. For measurement of velocity, as will be shown, we need a higher signal level. If the particles are smaller than the assumed $0.050 \mu \mathrm{m}$, which could easily be the case, the signal level would lower. Note that this signal level is lower than the signal level for molecular Rayleigh scattering from air at normal temperature and pressure. However, for a lower Mach number flow, the number density would be higher. As shown in figure 2, the number density is about an order of magnitude larger for Mach 2 flow than for Mach 4 flow.

\section{Fabry-Perot frequency discriminator}

The velocity is determined from the scattering light delivered to the optical processing system shown in figure 3 as follows.

Light exiting the fiber is collimated by lens L3 (145 mm focal length) and split into two paths with an uncoated optical flat (BS1). About $10 \%$ of the light is reflected and focused by lens L4 (85 mm focal length) onto PMT 1 (quantum efficiency $\sim 25 \%$ ). This signal is proportional to total scattered light. The light transmitted by the beamsplitter is directed through a planar mirror Fabry-Perot interferometer $(70 \mathrm{~mm}$ dia. mirrors, $90 \%$ reflectivity, $11 \mathrm{GHz}$ free spectral range (FSR), finesse 15). The light exiting the interferometer is focused by the fringe forming lens, L6. This lens consists of a pair of lenses (f/2 $135 \mathrm{~mm}$ focal length and $\mathrm{f} / 1.250 \mathrm{~mm}$ focal length) that has an effective focal length of $1600 \mathrm{~mm}$.

At the focal plane of the fringe pattern, the light is divided into two parts by a pair of mirrors (image dissector). A small mirror (6 $\mathrm{mm} \mathrm{dia.)} \mathrm{is}$ centered on the fringe pattern and directs light from the central part of the fringe through lens L7 (100 mm focal length) to PMT 2. This small mirror is mounted on a larger mirror (25 $\mathrm{mm}$ dia.) that directs light from the outer part of the fringe through L8 (100 $\mathrm{mm}$ focal length) to PMT 3. The mirrors are tilted $\pm 3^{\circ}$ with respect to the optical axis. A typical calculated image of the inner fringe of Rayleigh scattered light is shown in figure 4. Note that a flow in the direction of the $\mathbf{K}$ vector, which here is in the flow direction, results in a positive frequency shift and increasing fringe diameter, while a flow in the direction opposite the $\mathbf{K}$ vector results in a negative frequency shift and decreasing fringe diameter. Thus, as the frequency of the Rayleigh scattered light increases, less light is detected by PMT 2 and more light is detected by PMT 3. At the mirror location, the diameter of the image of the optical fiber is $11 \mathrm{~mm}$.

Additional optics were included to provide a reference image of light at the unshifted laser frequency. To accomplish this, several components were temporarily placed in the optical path using remotely controlled pneumatic actuators. When placed in the beam path, a mirror directed laser light onto a diffuser that scattered light into the optical fiber. Also, a prism assembly (PA) was placed in the light path between the Fabry-Perot interferometer and the fringe forming lens. This served to direct light into a standard video camera. The video signal from this camera was digitized by a PC frame grabber card. A computer program analyzed this image and generated signals to control the FabryPerot mirror alignment. The computer data acquisition could be operated from two locations, one in the SWT control room, and the other at the remote location of the Fabry-Perot interferometer.

\section{Lower bounds for velocity measurement uncertainty}

Estimates of the measurement uncertainty for the technique described here, where the scattered light is analyzed with a planar mirror Fabry-Perot interferometer, are obtained by numerically calculating the Cramer-Rao lower bound ${ }^{8}$. The variance of the estimate of a parameter $\alpha_{i}$ is given by

$$
V\left(\alpha_{i}\right)=\left[\Gamma^{-1}\right]_{i i}
$$

where no summation over repeated indices is implied. For Poisson statistics, $\Gamma$ is the Fisher information matrix with elements

$$
\Gamma_{i j}=\sum_{q} \frac{1}{\left\langle N_{D_{q}}\right\rangle} \frac{\partial\left\langle N_{D_{q}}\right\rangle}{\partial \alpha_{i}} \frac{\partial\left\langle N_{D_{q}}\right\rangle}{\partial \alpha_{j}}
$$


where $\left\langle N_{D_{q}}\right\rangle$ is the expected number of counts from the $\mathrm{q}^{\text {th }}$ photodetector. The summation is over the number of photodetectors, and the quantities $\left\langle N_{D_{q}}\right\rangle$ depend on the details of the optical system and the flow parameters. We can calculate the Cramer-Rao lower bounds given by equation 5 for velocity measurements based on the optical configuration described in the previous section. This allows us to conduct parametric studies to determine the optimum configuration for the FabryPerot interferometer and for the light detection system. The expected number of photons detected in time interval $\Delta t$ by detector 1 (PMT 1 ) is simply $R N_{R} \Delta t$ where $R$ is the reflectivity of the beamsplitter BS1 and $N_{R}$ is given by equation 3. The expected number of photons detected by PMT 2 and PMT 3 are

$$
<N_{D q}>=(1-R) N_{R} \Delta t \iint S_{R}(f) I_{F P}\left(f, \theta_{r}\right) f_{L}^{2} d f d A
$$

where the integrations are over frequency and the area of the $q^{\text {th }}$ detector (i.e., PMT 2 or 3 ), $f_{L}$ is the focal length of the fringe forming lens, and $I_{F P}$ is the FabryPerot instrument function given by

$$
I_{F P}(\psi)=\left[1+F \sin ^{2}\left(\frac{\psi}{2}\right)\right]^{-1}
$$

where $\psi$ is the phase change (neglecting any phase change on reflection) of the light between successive reflections given by

$$
\psi\left(f, \theta_{r}\right)=\frac{4 \pi f \mu d \cos \theta_{r}}{c}
$$

Here, $\mu$ is the refractive index of the medium in the Fabry-Perot cavity, $d$ is the Fabry-Perot mirror spacing, $\theta_{r}$ is the angle between the ray and the optic axis, and $F=1 /\left(\sin ^{2}\left(\pi / 2 N_{E}\right)\right.$ where $N_{E}$ is the effective finesse. In general, the image of a monochromatic extended source located in the object plane consists of a series of unequally spaced concentric rings. In this work, however, the field of view is restricted by the diameter of the optical fiber and includes only the inner fringe as shown in figure 4.

We can readily calculate the lower bounds for velocity measurement uncertainty based on the detected light using three PMT's as shown in figure 3. The uncertainties are evaluated as a function of the zero velocity fringe order $n_{o}$ and the measured component of the flow velocity $u_{K}$.

It is convenient to describe the fringe location in terms of the fringe order rather than fringe radius because of the nonlinear nature of the spectral response of the
Fabry-Perot. For example, if there is a bright fringe on axis, concentric bright fringes occur at integral values of lower orders, but the change in fringe radius decreases with decreasing fringe order.

Here we define the fractional order of the fringe with radius $r$ as

$$
n=\frac{d}{\lambda}\left(\frac{r}{f_{L}}\right)^{2}
$$

For example, at the location of the image dissector, the fringe order of the $6 \mathrm{~mm}$ diameter mirror is 0.10 . Likewise, the fringe order corresponding to the diameter of the image of the optical fiber is 0.33 . And, if unshifted laser light generates a bright fringe with radius $r_{o}$, we refer to this as order $n_{o}$. Note that here we are using the term "fringe order" to denote change of the fringe order from the actual fringe order on the optical axis, which is $2 d / \lambda$.

frequency is

The change in fringe order with optical

$$
\Delta n=\frac{\Delta f}{F S R}
$$

so the change of fringe order $\Delta n$ with change of velocity $\Delta V$ is given by

$$
\Delta n=\frac{(2 / \lambda) \sin \left(\theta_{s} / 2\right)}{F S R} \Delta V
$$

where $\theta_{s}$ is the scattering angle. For example, with a velocity of $100 \mathrm{~m} / \mathrm{sec}$, a $F S R$ of $11 \mathrm{GHz}$ and $120^{\circ}$ degree scattering, the change in the fringe order $\Delta n$ is 0.0296 .

Figure 5a shows the amounts of light detected from the inner and outer regions of the fringe pattern as a function of velocity for a FSR of $11 \mathrm{GHz}$, and for $n_{0}=$ 0.1 . Figure $5 b$ shows the relation between the ratio of the outer/inner counts as a function of velocity. Note that this quantity is independent of the intensity of the scattered light. Also note that this is a single valued function only for velocities with a magnitude less than $300 \mathrm{~m} / \mathrm{sec}$. The lower bound for velocity uncertainty is shown in figure 5c. This shows that the uncertainty is less than $6 \mathrm{~m} / \mathrm{sec}$ for velocity magnitudes less than about $175 \mathrm{~m} / \mathrm{sec}$. It should be pointed out that the minimum uncertainty value can be located at any given velocity by shifting the value of $n_{0}$. Thus the important information given by this analysis is that the velocity measurement uncertainty is less than $6 \mathrm{~m} / \mathrm{sec}$ over a $350 \mathrm{~m} / \mathrm{sec}$ range.

We can also examine the effect of changing the system parameters. For example, figure 6 shows the velocity uncertainty for several values of the interferometer free spectral range. This shows that a tradeoff exists between uncertainty and range. A smaller FSR gives lower uncertainty, but over a smaller range 
than higher values of $F S R$. The results presented in this paper were taken with $F S R=11 \mathrm{GHz}$.

\section{EXPERIMENT}

An experiment was conducted to evaluate the technique described above for measuring dynamic velocity. The GRC 10x25 cm supersonic wind tunnel was selected for these tests. The currently installed Mach 4 nozzle blocks were used for convenience, although this presented a much more demanding measurement situation because of the low density. Flows in PDE tests will generally be run at higher densities.

\section{Supersonic wind tunnel}

The GRC $10 \times 25 \mathrm{~cm}$ continuous flow supersonic wind tunnel, configured for Mach 4 flow in the test section, is shown in figure 7. The air is supplied from the GRC laboratory-wide central compressed air system at pressures up to about $45 \mathrm{psia}$. The amount of water vapor in the air supply depended on the particular equipment (compressors, chillers, dryers) in use for the test. The flow from the tunnel exits into the laboratory exhaust system, which can reach pressures of less than 0.5 psia. The mass flow for our tests was typically about $1 \mathrm{~kg} / \mathrm{sec}$. An electrical heater mounted in the tunnel air supply line could be used to raise the temperature of the air supplied to the tunnel. For these tests, the air temperature was maintained at $25^{\circ} \mathrm{C}$. A $0.2 \mu \mathrm{m}$ filter was located in the air supply line to remove particulates. Optical access was provided by float plate glass windows $(51 \mathrm{~cm}$ long, $25 \mathrm{~cm}$ high, $4.8 \mathrm{~cm}$ thick) mounted in the tunnel.

A set of spray nozzles was located $3 \mathrm{~m}$ upstream of the tunnel plenum to provide a capability to inject water aerosol to raise the humidity level and thus increase the quantity of scattering centers.

An existing wedge-shaped supersonic injector mounted in the floor of the SWT test section was used to generate a moving shock wave. When the injector was pressurized, the resulting nozzle plume generated a bow shock, which moved through the probe volume as illustrated in figure 8 .

A $3 \times 3$ set of optical ports on $50.8 \mathrm{~mm}$ centers was located in the tunnel wall, as shown in figures 7 and 9. A baffle assembly, designed to allow the laser beam to pass into and out of the test section without scattering laser light in the field of view of the receiving optics, could be placed in one of these ports. The $30 \mathrm{~cm}$ long assembly had a $19 \mathrm{~mm}$ thick window that provided the pressure seal. Several baffles inside the tube, as shown on figure 7 , were used to control the laser light scattered by the window. The beam then entered the tunnel through a final $3.2 \mathrm{~mm}$ diameter aperture located flush with the inside of the tunnel. A second similar tube was mounted on the opposite tunnel wall and served to capture the laser beam and minimize light scattered back into the tunnel.

\section{Optical configuration}

Light from a $5 \mathrm{~W}, 532 \mathrm{~nm}$, single-frequency, $\mathrm{Nd}$ :Vanadate CW laser was focused by a $1000 \mathrm{~mm}$ focal length lens to a $200 \mu \mathrm{m}$ diameter beam at the probe volume located in the wind tunnel test section. Scattered light was collected from a downstream location at a $120^{\circ}$ scattering angle and focused by a pair of $250 \mathrm{~mm}$ focal length lenses into a $0.55 \mathrm{~mm}$ core diameter, $0.22 \mathrm{NA}, 130 \mathrm{~m}$ long optical fiber. The fiber was routed from the high-noise test cell to a quiet remote location, which provided a stable environment for the Fabry-Perot interferometer based optical processing system . This optical arrangement resulted in the measured velocity component being at $63.4^{\circ}$ from the tunnel axis. The length of the probe volume was about $1.2 \mathrm{~mm}$. Assuming that the flow is along the tunnel axis, the measured component $u_{K}=0.447 V_{a}$, where $V_{a}$ is the tunnel flow velocity.

\section{Data acquisition}

The photoelectron pulses from the three PMT's were amplified (Gain $=5$ ) and sent to constant fraction discriminators (CFD). The $10 \mathrm{~ns}$ wide TTL level pulses from the CFD's output were counted by a PC countertimer board. Pulses could be simultaneously counted on the three channels at rates to $80 \mathrm{MHz}$ indefinitely. Photoelectron count rates encountered in this work were up to $30 \mathrm{MHz}$. The accumulated counts on each channel were recorded at preset time intervals, typically 10 to $1000 \mu \mathrm{sec}$, and stored on the computer hard disk. The number of counts in each time interval was given by the difference between adjacent values of the accumulated counts.

\section{Data processing}

The total light scattering is measured by PMT 1. One approach to processing the PMT signals in order to obtain the velocity would be to use parameter estimation techniques based on the model developed for the calculation of the lower bounds of measurement uncertainty. This would entail doing a nonlinear fit of the data to the model function given by equation 7 . However, because of the large quantity of data a simpler procedure was used. A linear relation was assumed between the ratio of the signals from the outer and inner regions (PMT's 2 and 3). The tunnel flow velocity 
measured using wall static pressure near the probe volume was used as a calibration point. The resulting relation between ratio of outer/inner counts to tunnel axial velocity is shown in figure 10 .

\section{Results}

Data were obtained at two stations within the tunnel. Station 1 was 1.5 in $(38 \mathrm{~mm})$ downstream from the leading edge of the wedge-shaped injector at a height of 0.75 in $(19 \mathrm{~mm})$ above the test section floor. Station 2 was 3.5 in $(89 \mathrm{~mm})$ downstream from the leading edge at a height of 2.75 in $(70 \mathrm{~mm})$ above the tunnel floor.

Figure 11a shows a time record of three channels of data obtained at Station 1. Data was acquired at a $1 \mathrm{kHz}$ sampling rate for 10 seconds. The axial tunnel velocity calculated using the linear relation between ratio of outer/inner signals and velocity is shown in figure $11 \mathrm{~b}$. At the time the injector was pressured, the signal total signal level dropped from about 30 million counts/sec to 1.3 million counts/sec. This is probably due to the clean air from the injector entering the probe volume in place of the tunnel flow with its relatively large number density of scattering centers. The rms fluctuation in the measured velocity is about $28 \mathrm{~m} / \mathrm{sec}$ or $4 \%$. This can be due to either actual flow velocity fluctuations, shot noise, or some combination of both. The velocity measured in the time after the injector was pressurized exhibited much larger velocity fluctuations as a result of the much lower signal levels.

An example of data taken at Station 2 is shown in figure 12. Data were acquired at a $1 \mathrm{kHz}$ sampling rate for 30 seconds. At this location, as opposed to the data taken at Station 1, the level of the signal increased slightly as the injector was pressurized. Because the total signal level ( 7 million counts/sec without injection, 8.5 million counts/sec with injection) did not change significantly, the rms fluctuation in the measured velocity was about the same $(50 \mathrm{~m} / \mathrm{sec})$ for both states. This probe volume location appeared to be below and downstream of the bow shock, but not in the dry air of the injector plume. Note total count rate for this data was only about $25 \%$ of the count rate for the data taken at Station 1. This is probably due to the difference in the humidity level of the supply air on the two days.

\section{CONCLUSIONS}

The results of this initial study demonstrated a technique using a Fabry-Perot interferometer as an optical frequency discriminator to measure flow velocity at a point at sampling rates up to $100 \mathrm{kHz}$. However, obtaining acceptable measurement uncertainty is limited by the quantity of scattering centers in the flow. High frequency response measurements require high seeding levels. The measurements reported here for Mach 4 flow, where condensation of water vapor in the air supply provided the seeding, did not have sufficient strong scattering to give acceptable measurement uncertainties at the desired $100 \mathrm{kHz}$ sampling rates. The introduction of additional moisture using water mist injection did not significantly increase signal levels. For lower Mach number expansion, the seeding levels would be significantly higher. For example, Mach 2 expansion would provide an order of magnitude higher signal level. Additional water vapor could also be added to the flow by heating the supply air and using the water mist injection system.

Other flow experiments will need seed material provided by an aerosol generator. However, it may be difficult to use standard smoke generators to introduce seed in the high pressure flow found in many experiments.

Signal levels can also be increased by use of higher power lasers, higher efficiency light collection optics and larger probe volumes. It should be noted that although photon counting electronics were used in this study, photon counting is limited to a maximum of about $50 \mathrm{MHz}$ because of pulse pile-up errors. At higher signal levels, analog data acquisition should be used. Also, for higher signal levels, it may be advantageous to use high quantum efficiency semiconductor detectors instead of the PMT's used here.

Another approach for obtaining high sampling rate transient measurements is to use a pulse burst laser system that can provide up to a $\mathrm{MHz}$ pulse rate for a short time (up to about 100 pulses).

\section{REFERENCES}

${ }^{1}$ Smeets, G., and A. George, "Michelson spectrometer for instantaneous Doppler velocity measurements", J. Phys. E: Sci. Instrum, 14, pp. 838-845,1981.

2 Jackson, D.A, and D.M. Paul, "Measurement of supersonic velocity and turbulence by laser anemometry", J. Phys. E: Sci. Instrum, 4, pp. 173 177, 1971.

${ }^{3}$ Korb, C.L., B.M. Gentry and C.Y. Weng, "Edge technique: theory and application to the lidar measurement of atmospheric wind", Appl. Opt. 31, pp. 4202-4213, 1992 .

${ }^{4}$ Crafton, J., N.M. Messersmith and J.P. Sullivan, "Filtered Doppler velocimetry: Development of a point system", AIAA paper $98-0509$, Reno $36^{\text {th }}$, Jan 12-15, 1998. 
${ }^{5}$ Kuhlman, J., P. Collins, and T. Scarberry, "Twocomponent point Doppler velocimetry data in circular jets," Meas. Sci. Technol., 12, pp. 395408, 2001.

${ }^{6}$ Seasholtz, R.G., J. Panda, and K.A. Elam, "Rayleigh scattering diagnostic for dynamic measurement of velocity fluctuations in high speed jets", AIAA $39^{\text {th }}$ Aerospace Sciences Meeting, Reno, NV, AIAA2001-0847, 2001.

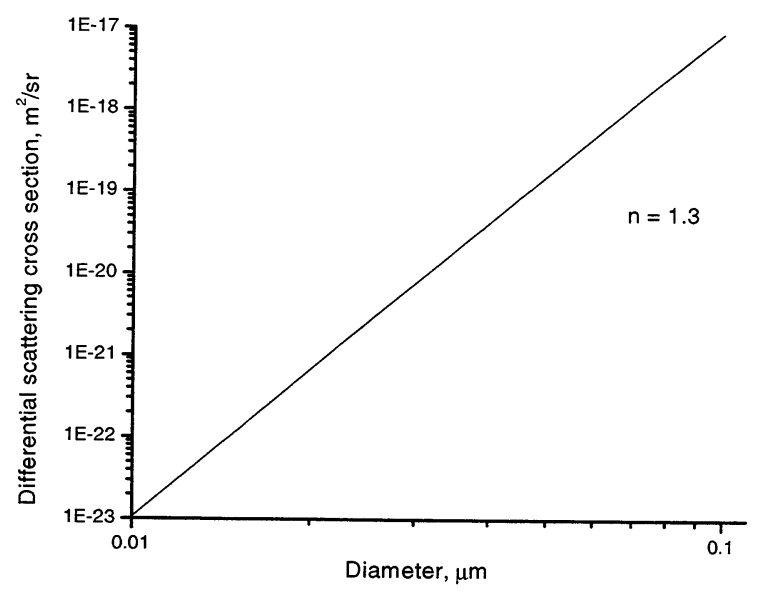

Fig. 1.-Differential scattering cross section for scattering of $532 \mathrm{~nm}$ light from water droplets.
${ }^{7}$ Lai, D.S., Generation of Monodisperse Droplets by Spontaneous Condensation of Flow in Nozzles, Ph.D. Thesis, Case Western Reserve University, Cleveland, OH, 1993. [Also, NASA Grant NAG3831 Final Report].

${ }^{8}$ Whalen, A.D., Detection of Signals in Noise, Academic Press, New York, pp. 324-231, 1971.

${ }^{9}$ Vaughan, J.M., The Fabry Perot Interferometer, History, Theory, Practice and Applications, Adam Hilger, Bristol, pp. 89-112, 1989.

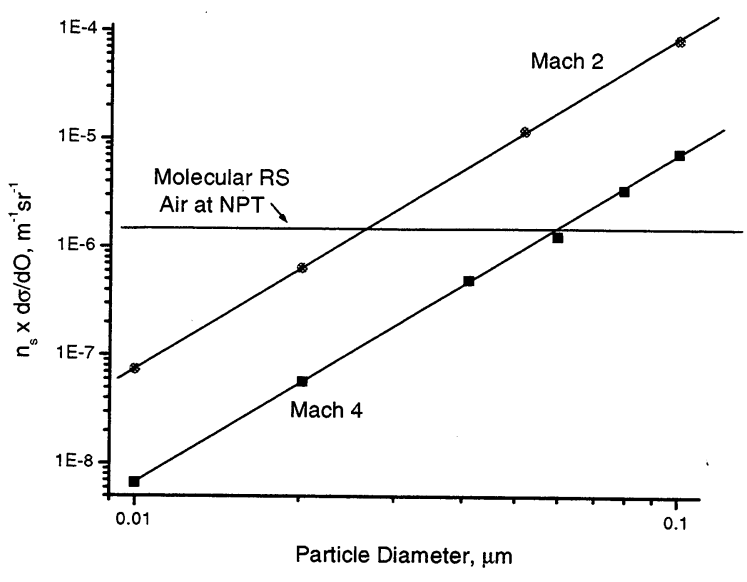

Fig. 2.-Product of number density and differential scattering cross section for scattering from condensed water vapor.

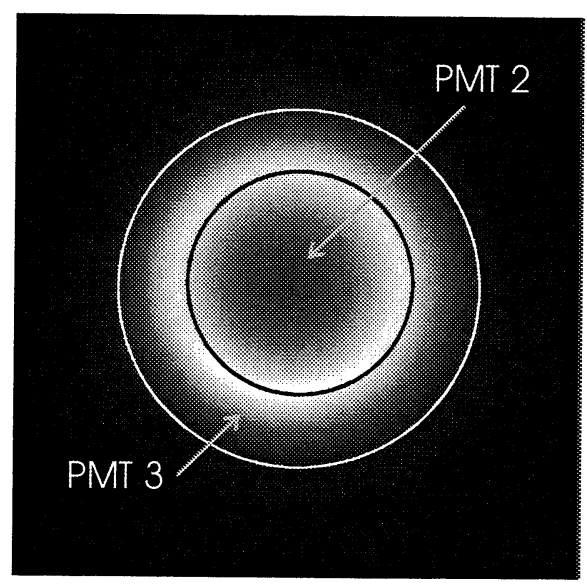

Fig. 4.-Fabry-Perot interferometer fringe showing two regions where light is directed to PMT 2 (INNER) and PMT 3 (OUTER). 

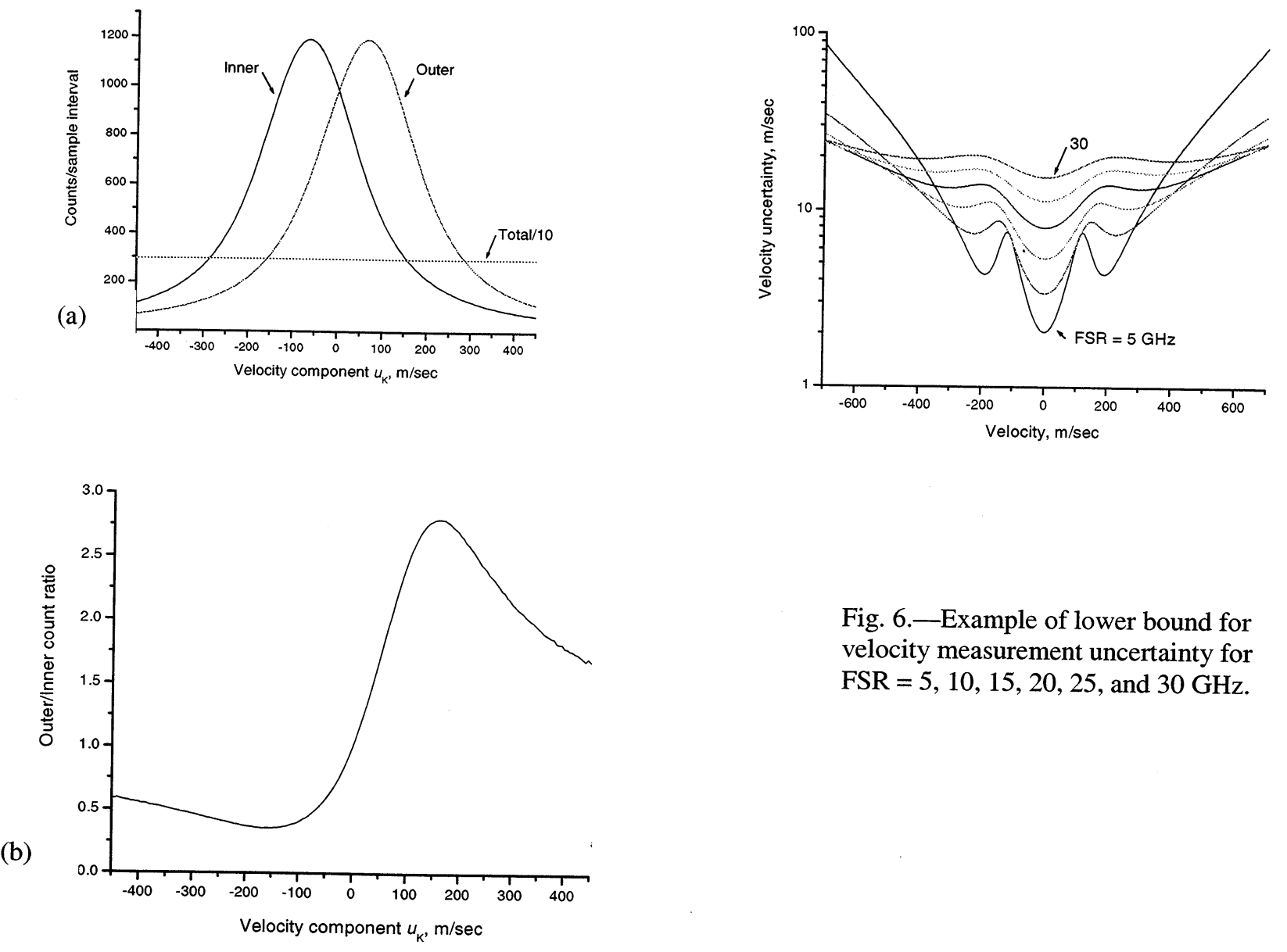

Fig. 6.-Example of lower bound for velocity measurement uncertainty for $\mathrm{FSR}=5,10,15,20,25$, and $30 \mathrm{GHz}$.

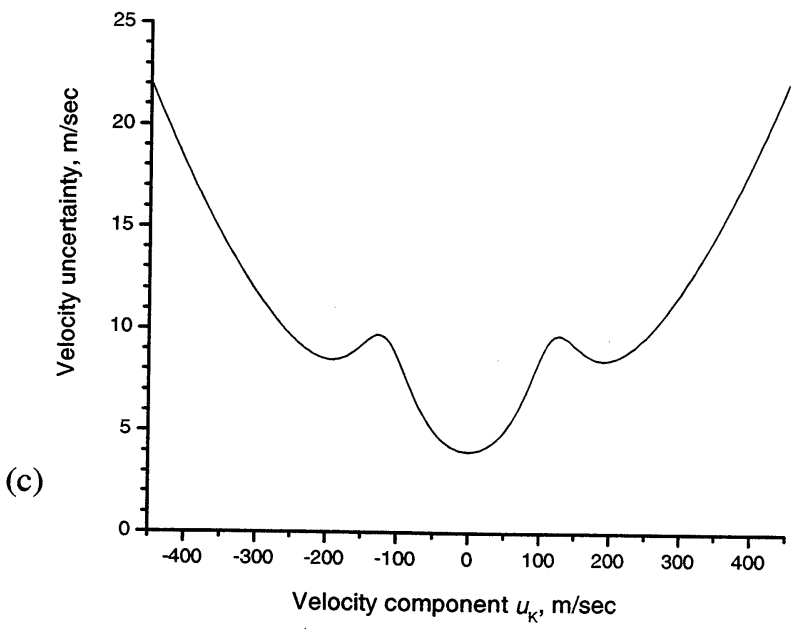

Fig. 5.-Determination of velocity from scattering of $532 \mathrm{~nm}$ laser light from condensate fog in Mach 4 flow. (a) signals from three PMT's; (b) ratio of outer/inner counts as function of measured velocity component; (c) lower bound for estimate of velocity measurement uncertainty. 
Supersonic Wind Tunnel With Various Configurations of Point Measurement System Using Supersonic Injection, Created Bow Shock Evaluation

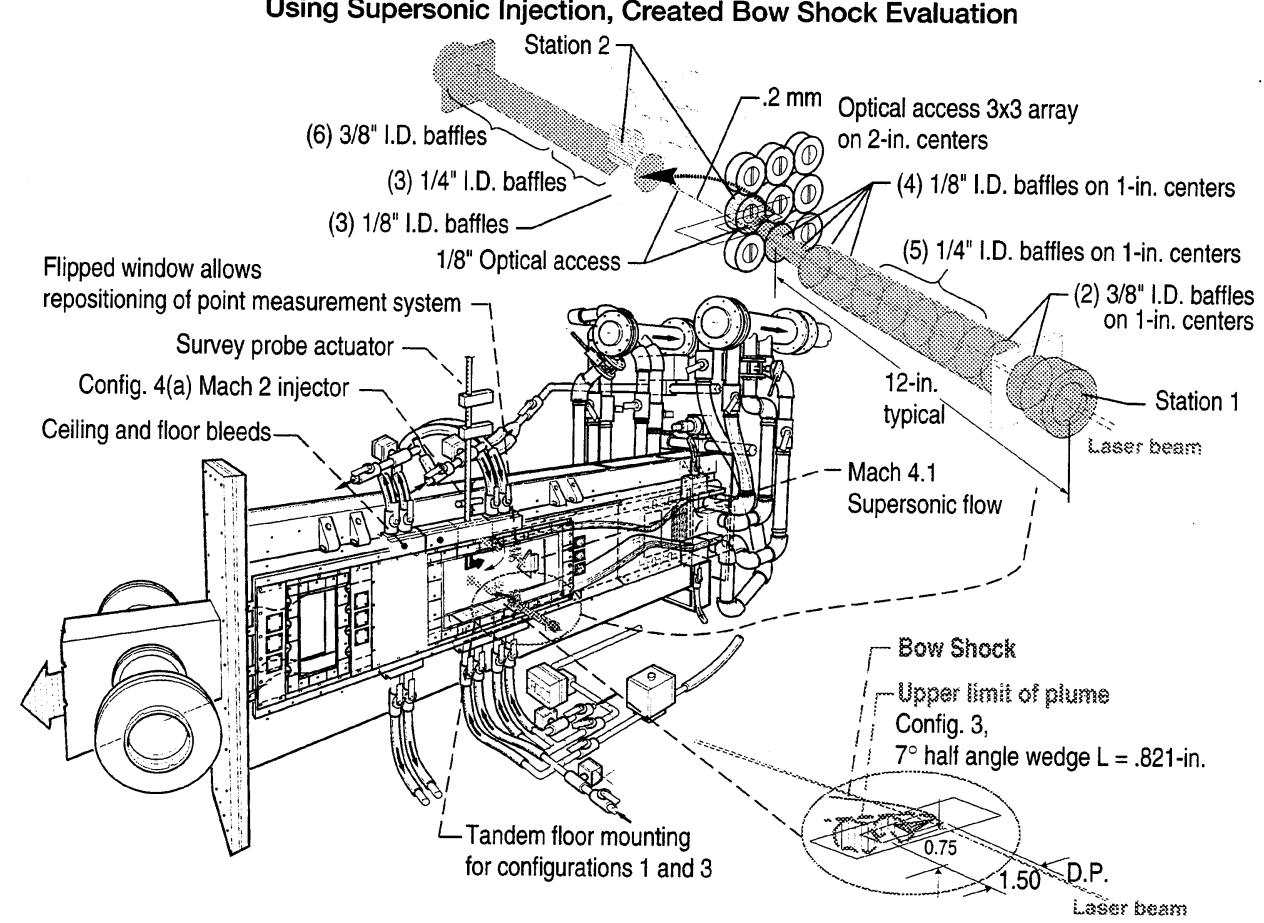

Fig. 7.-Glenn Research Center 10 x 25 supersonic wind tunnel.

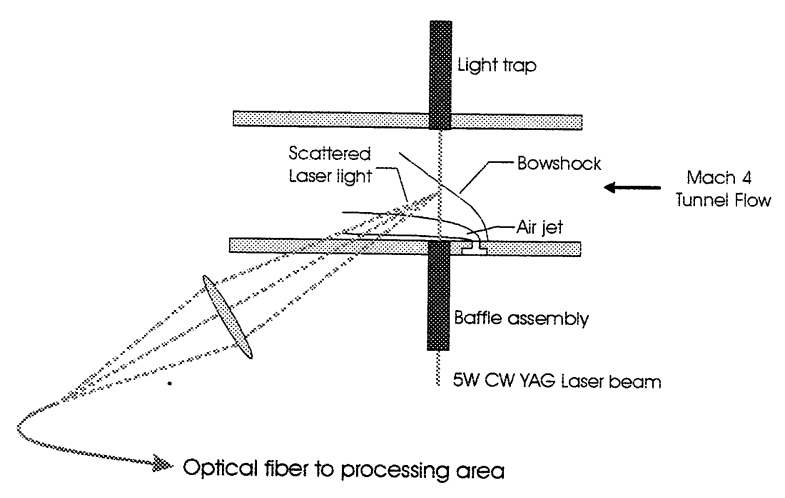

Fig. 8.-Schematic view of bow shock generated by air injection from supersonic wedge shaped nozzle into supersonic cross flow.

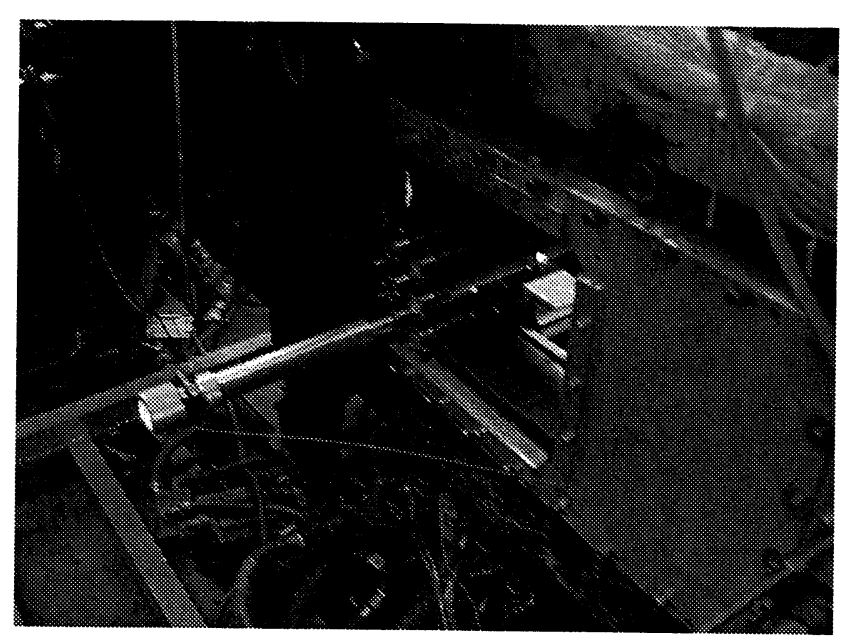

Fig. 9.-Photograph of baffle assembly used to reduce stray laser light scattering. 


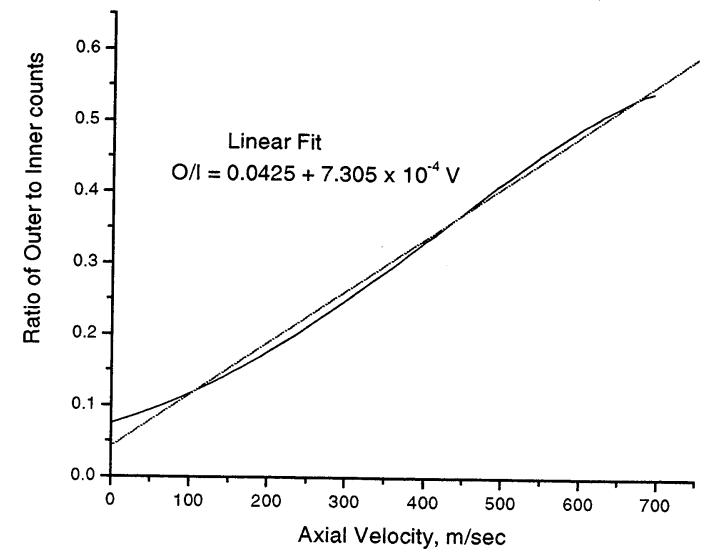

Fig. 10.-Linear calibration of ratio of outer/inner counts as function of SWT axial . velocity.

(a)

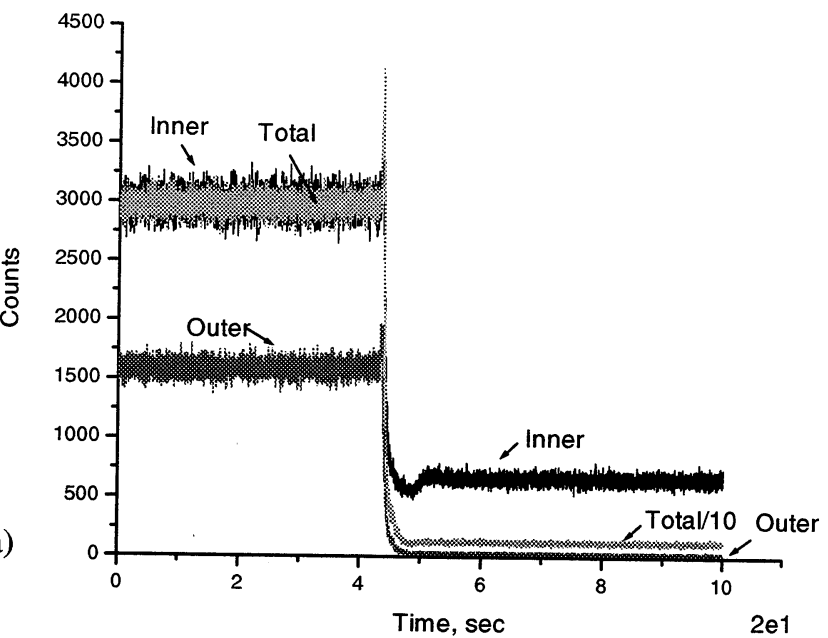

(b)

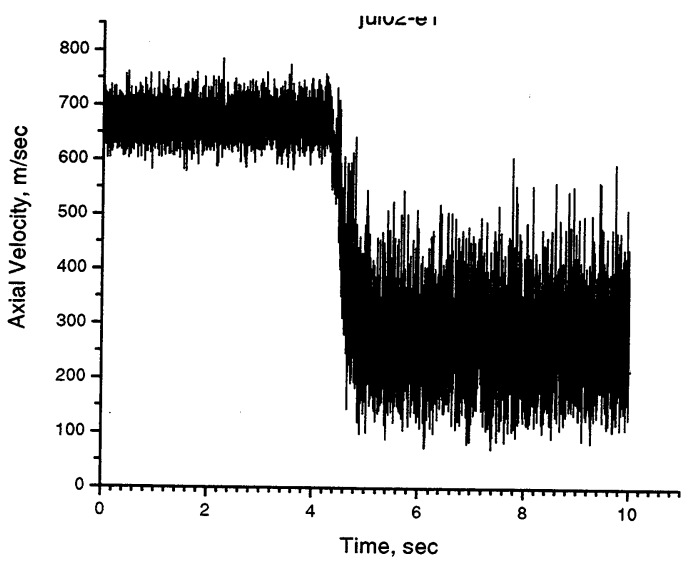

Fig. 11.-Data from Station 1 as injector was pressurized. (a) PMT signals; (b) SWT axial velocity.

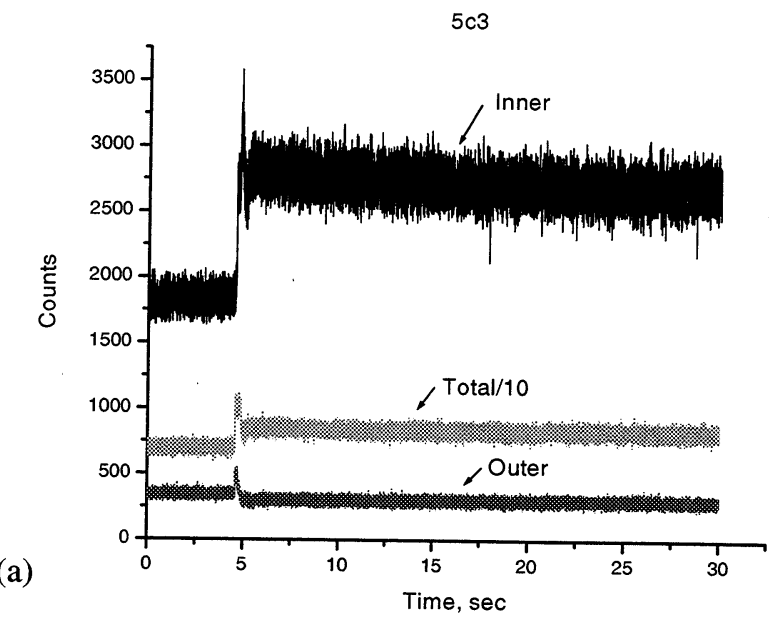

(b)

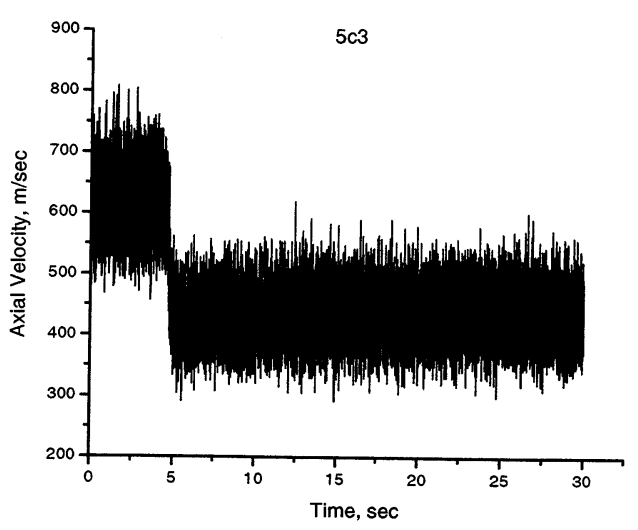

Fig. 12.-Data from Station 2 as injector was pressurized. (a) PMT signals; (b) SWT axial velocity. 


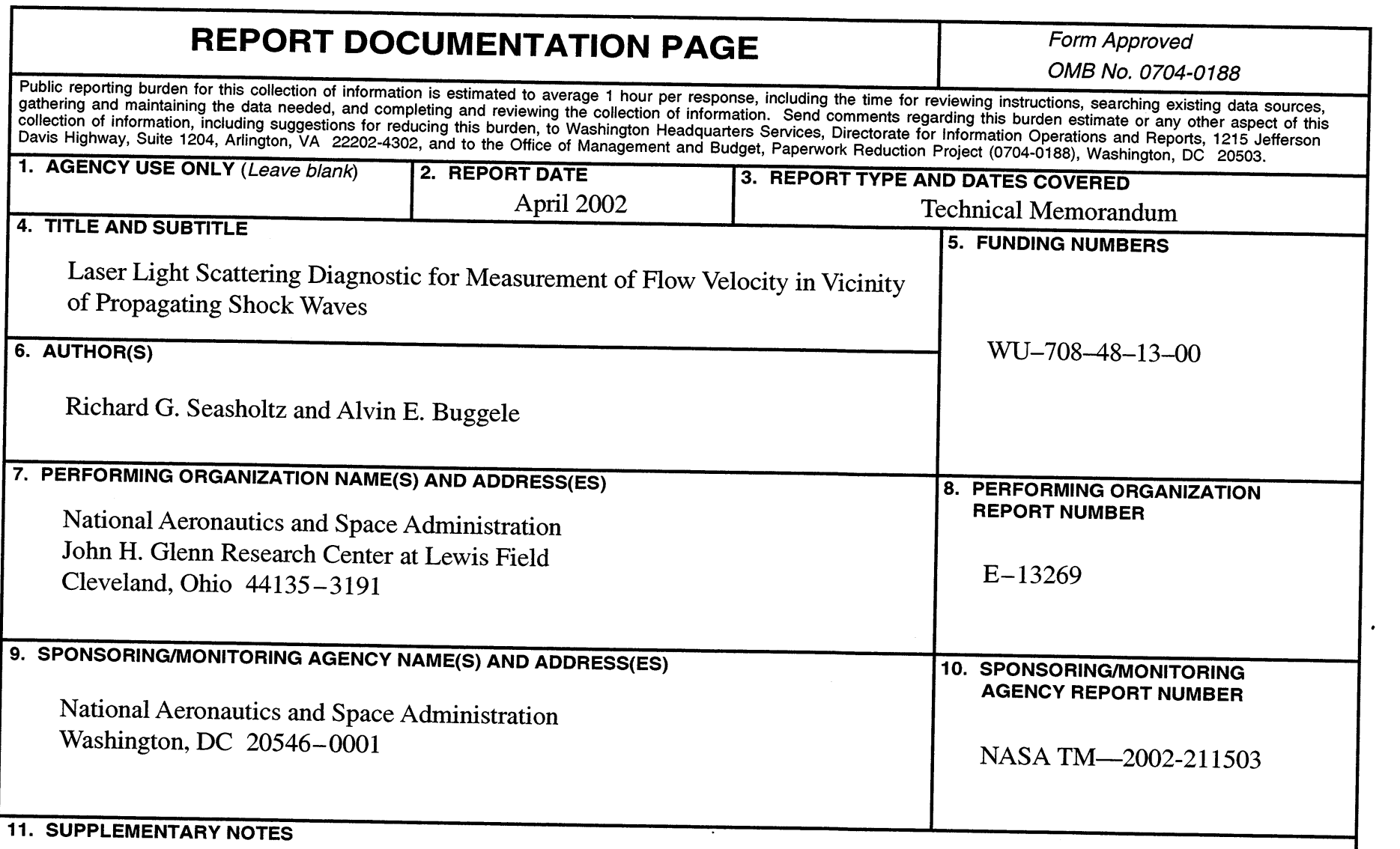

Prepared for the 2001 19th International Congress on Instrumentation in Aerospace Simulation Facilities (ICIASF 2001) cosponsored by the IEEE AES, NASA Glenn, and OAI, Cleveland, Ohio, August 27-30, 2001. Responsible person,
Richard Seasholtz, organization code 5520, 216-433-3754.

12a. DISTRIBUTION/AVAILABILITY STATEMENT

Unclassified - Unlimited

Subject Category: 35

Distribution: Nonstandard

Available electronically at http://gltrs.grc.nasa.gov/GLTRS

This publication is available from the NASA Center for AeroSpace Information, 301-621-0390.

13. ABSTRACT (Maximum 200 words)

A laser light scattering diagnostic for measurement of dynamic flow velocity at a point is described. The instrument is being developed for use in the study of propagating shock waves and detonation waves in pulse detonation engines under development at the NASA Glenn Research Center (GRC). The approach uses a Fabry-Perot interferometer to measure the Doppler shift of laser light scattered from small (submicron) particles in the flow. The high-speed detection system required to resolve the transient response as a shock wave crosses the probe volume uses fast response photodetectors and a PC based data acquisition system. Preliminary results of measurements made in the GRC Mach 4,10 by $25 \mathrm{~cm}$ supersonic wind tunnel are presented. Spontaneous condensation of water vapor in the flow is used as seed. The tunnel is supplied with continuous air flow at up to $45 \mathrm{psia}$ and the flow is exhausted into the GRC laboratory-wide altitude exhaust system at pressures down to 0.3 psia.

\section{SUBJECT TERMS}

Rayleigh scattering; Fabry-Perot interferometers

\begin{tabular}{|l|l|} 
& $\begin{array}{c}\text { 15. NUMBER OF PAGES } \\
16\end{array}$ \\
\cline { 2 - 3 } & 16. PRICE CODE \\
\hline $\begin{array}{l}\text { 19. SECURITY CLASSIFICATION } \\
\text { OF ABSTRACT } \\
\text { Unclassified }\end{array}$ & 20. LIMITATION OF ABSTRACT \\
\hline \multicolumn{2}{|l|}{$\begin{array}{l}\text { Standard Form 298 (Rev. 2-89) } \\
\text { Prescribed by ANSI Std. Z39-18 } \\
298-102\end{array}$}
\end{tabular}

\begin{tabular}{|c|c|}
\hline $\begin{array}{c}\text { 17. SECURITY CLASSIFICATION } \\
\text { OF REPORT } \\
\text { Unclassified }\end{array}$ & $\begin{array}{c}\text { 18. SECURITY CLASSIFICATION } \\
\text { OF THIS PAGE } \\
\text { Unclassified }\end{array}$ \\
\hline
\end{tabular}

